\title{
Intervenciones psicológicas en adultos con discapacidad intelectual y trastorno límite de la personalidad comórbido
}

\author{
Psychological interventions in adults with intellectual disabilities and \\ comorbid personality limit disorder
}

\begin{abstract}
Resumen
Objetivo: la importancia de identificar la comorbilidad psicopatológica permite responder de forma más efectiva a la aplicación de ciertos tratamientos específicos, en función de las características de los individuos con discapacidad intelectual y su perfil psicopatológico. El objetivo de la presente revisión es describir cuáles son las intervenciones que se llevan a cabo en individuos adultos con discapacidad intelectual asociada a un trastorno límite de la personalidad (TLP) y determinar su efectividad. Método: en la revisión de la literatura se han identificado 24 artículos, 11 hacen referencia a la prevalencia de los trastornos psiquiátricos en individuos con discapacidad intelectual y las implicaciones que supone, y 13 a las intervenciones psicológicas en el ámbito de la discapacidad intelectual y TLP comórbido. Las bases de datos utilizadas son WOS (Web of Science), SCOPUS, CINALH, PsycINFO, LILACS y la librería Cochrane. Resultados: el enfoque psicoterapéutico en población con discapacidad intelectual es controvertido y la evidencia sobre las terapias psicológicas es limitada. Respecto a las intervenciones utilizadas, la que aporta unos resultados favorables para la reducción de la sintomatología es la terapia dialéctica conductual.
\end{abstract}

\section{Palabras clave}

Intervención o tratamiento, trastorno de la personalidad, discapacidad intelectual.

\begin{abstract}
Objective: an accurate diagnosis of psychopathological disorders allows us to respond more effectively with the application of specific treatments, taking into account the individual features of intellectual disability and the psychopathological profile of the patient. The aim of this review is to describe the types of interventions developed to adults with intellectual disabilities and comorbid personality limit disorder. Method: in the review of the literature, 24 articles have been identified, 11 refer to the prevalence of psychiatric disorders in individuals with intellectual disabilities and the implications involved, and 13 to psychological interventions in the field of intellectual disability and comorbid personality limit disorder. The databases used are WOS (Web of Science), SCOPUS, CINALH, PsycINFO, LILACS and the Cochrane library. Results: the psychotherapeutic approach in population with intellectual disabilities is controversial and the evidence on psychological therapies is limited. Regarding the interventions used, the one that provides favorable results for the reduction of the symptomatology is the dialectic behavioral therapy.
\end{abstract}

\section{Keywords}

Intervention or treatment, borderline personality disorders, intellectual disability.

\section{Dafne Galí Rapún \\ <rapun.gali.dafne@gmail.com>}

Universidad Autónoma de Barcelona. España

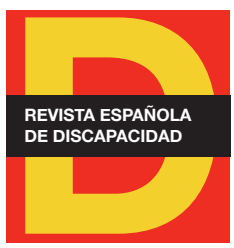

Para citar:

Galí, D. (2018): "Intervenciones psicológicas en adultos con discapacidad intelectual y trastorno límite de la personalidad comórbido". Revista Española de Discapacidad, 6 (II): 49-62.

Doi: <https://doi.org/10.5569/23405104.06.02.03>

Fecha de recepción: 03-03-2017 Fecha de aceptación: 26-06-2018

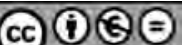




\section{Introducción ${ }^{1}$}

El término discapacidad intelectual ha sufrido ciertos cambios terminológicos, así como la definición utilizada para describir de forma precisa el término y establecer el significado y sus límites. En primer lugar, la idea de retraso mental ha sido sustituida por discapacidad intelectual debido a que no comunica dignidad o respeto y da lugar a la devaluación de estas personas (Schalock et al., 2007); y en segundo lugar, la concepción de discapacidad intelectual implica un constructo incluido dentro del constructo general de discapacidad y una visión más completa del individuo incluyendo aspectos biológicos y sociales, y reconociendo la multidimensionalidad del ser humano (Schalock et al., 2007). Una de las muchas definiciones que engloba las características citadas anteriormente, define la discapacidad intelectual como las limitaciones significativas tanto en el funcionamiento intelectual como en la conducta adaptativa tal y como se ha manifestado en habilidades conceptuales, sociales y prácticas. Esta discapacidad se origina antes de los 18 años (Asociación Americana de Discapacidades Intelectuales y del Desarrollo AAIDD, 2011). Teniendo en cuenta las dificultades que conlleva la discapacidad intelectual, la comorbilidad con algunos trastornos psicológicos es una complicación común, creando así problemas significativos en diferentes ámbitos de la vida de estos individuos (Turygin et al., 2014). Algunos estudios destacan las altas tasas de comorbilidad en esta población respecto a aquella que no presenta discapacidad intelectual (Wieland et al., 2014; Martorell et al., 2009; Di Nuovo y Buono, 2007; Whitaker y Read, 2006), estimándose que la presencia de un trastorno mental en individuos con discapacidad intelectual es hasta 4 o 5 veces mayor respecto a la población general (Rush et al., 2004). Según el 1999 Surgeon General's Report on mental health (USA), se estima que el $21 \%$ de los adultos en la población general presentan un trastorno mental, sin embargo, el porcentaje oscila entre $17 \%$ y $36 \%$ para aquellos adultos con discapacidad intelectual y diagnosticados con un trastorno mental (Kerker et al., 2004). Coincidiendo con las tasas de prevalencias anteriores se estima que entre el $10 \%$ y el $39 \%$ de individuos con discapacidad intelectual presenta un trastorno mental (Costello y Bouras, 2006). Aun así, en función de la gravedad de la discapacidad intelectual el proceso de diagnóstico de un trastorno mental puede verse más o menos afectado y las tasas de prevalencia pueden variar. Otra de las posibles explicaciones sobre las tasas tan elevadas de trastorno mental en dicha población es debido a la disminución de las capacidades de afrontamiento provocadas por las alteraciones cognitivas, comunicativas y adaptativas (Rush et al., 2004). Estas bajas capacidades de afrontamiento aumentan el riesgo de la aparición de psicopatología y problemas conductuales (Rush et al., 2004). Como se ha mencionado anteriormente, las tasas de prevalencia varían en función de la gravedad ya que los individuos con una discapacidad intelectual leve presentan menos dificultades comunicativas y son más conscientes de sus limitaciones. Es por ello que el proceso de diagnóstico es menos complejo cuando la gravedad es leve, a diferencia de cuando se trata de una discapacidad severa (Kerker et al., 2004).

Tras una breve descripción sobre la relación entre discapacidad intelectual y psicopatología en general, nos centraremos más concretamente en el trastorno límite de la personalidad (TLP). Dicho trastorno en este tipo de población se caracteriza por unas reacciones excesivas antes ciertas demandas, agresión verbal y física, apego excesivo respecto al personal cuidador y devaluación del resto, cambios de humor extremos como respuesta a eventos ambientales, incapacidad de relacionar dicho comportamiento con las conse-

1. La redacción del artículo se llevó a cabo como proyecto de final de Máster y posteriormente fue seleccionado para realizar una ponencia oral en el I Congreso de Psicología General Sanitaria celebrado en el Hospital del Mar (9,10 y 11 de junio de 2016). 
cuencias que conlleva, impulsividad y autolesión (Wilson, 2001). Dichas características no varían respecto a población general y la única diferencia radica que cuando se presenta comorbilidad en individuos con discapacidad intelectual la sintomatología se agrava. Los individuos con discapacidad intelectual no son inmunes de padecer un TLP aunque el proceso de diagnóstico es controvertido debido a las dificultades tanto cognitivas y comunicativas; aun así existe un consenso general respecto al incremento de la vulnerabilidad de estas personas a los trastornos de personalidad (Pridding y Procter, 2008). Al ser uno de los diagnósticos más difíciles, un individuo con discapacidad intelectual puede presentar mayoritariamente autolesiones, labilidad emocional, impulsividad y agresividad (Wilson, 2001). Las tasas de prevalencia oscilan entre el $1 \%$ y $91 \%$ a nivel comunitario y entre el $22 \%$ y el $92 \%$ en ambientes hospitalarios (Pridding y Procter, 2008). Estos datos ponen de manifiesto y cuestionan la utilidad clínica de un buen diagnóstico, por lo que se concluye que no existe una prevalencia exacta para los casos con discapacidad intelectual y un TLP (Pridding y Procter, 2008). A nivel terapéutico la intervención más efectiva para este tipo de población se basa en la terapia farmacológica, la psicoterapia y el manejo de la conducta. Cabe decir que la intervención farmacológica debe ir acompañada de estrategias conductuales y psicoterapéuticas para así aumentar su efectividad (Wilson, 2001).

La importancia de identificar la comorbilidad psicopatológica permite responder de forma más efectiva a la aplicación de ciertos tratamientos específicos en función de las características de los individuos con discapacidad intelectual y su perfil psicopatológico (Turygin et al., 2014), así como una adecuada planificación de los servicios de salud mental (Di Nuovo y Buono, 2007). En ocasiones este tipo de pacientes pasan desapercibidos o son considerados como un grupo invisible ante los servicios de salud mental (Wieland et al., 2015). Otra de las dificultades que se hace patente en los sistemas de salud mental es la falta de recursos, conocimientos o interés para satisfacer las necesidades de esta población (Rush et al., 2004). Este colectivo suele experimentar dificultades para acceder al sistema de salud mental, y poder beneficiarse de un tratamiento psicológico. En definitiva, es necesaria una adecuada formación específica de los profesionales en salud mental. Así como un aumento de la conciencia de que este colectivo requiere de una asistencia en servicios especializados, debido al nivel de complejidad que supone tener una discapacidad intelectual y un trastorno psicopatológico.

El objetivo de la presente revisión es describir cuáles son las intervenciones que se llevan a cabo en individuos adultos con discapacidad intelectual asociada a un trastorno límite de la personalidad y determinar la eficacia de las mismas.

\section{Método}

Se ha realizado una búsqueda bibliográfica basada en la literatura usando diversas bases de datos electrónicas como WOS (Web of Science), SCOPUS, CINALH, PSYCINFO, LILACS y la librería Cochrane. Debido a la poca cantidad de literatura relacionada con esta área, la búsqueda se ha complementado usando Google académico y la búsqueda manual de las referencias bibliográficas de los artículos seleccionados. Como criterios de inclusión, se han seleccionado artículos con una metodología cualitativa, escritos en lengua 
inglesa y desde 2001 a 2018; excepto un informe en español. Los términos de búsqueda empleados para cada base de datos han sido los siguientes: intervención o tratamiento psicológico, trastornos límite de la personalidad y discapacidad intelectual. Se han identificado 1231 artículos en las diferentes bases de datos citadas anteriormente combinando los diferentes términos de búsqueda. De cada uno de los artículos encontrados se ha realizado una lectura del resumen para determinar la utilidad en dicho estudio, solo se han incluido 58. De estos 58 artículos, 34 se han excluido por ser cuantitativos y únicamente se han incluido 24 por ser cualitativos y centrarse en trastorno límite de la personalidad y discapacidad intelectual. Del total de los 24, 11 hacen referencia a la prevalencia de los trastornos psiquiátricos en individuos con discapacidad intelectual y las implicaciones que supone y 13 a las intervenciones psicológicas en el ámbito de la discapacidad intelectual y TLP comórbido. Se han incluido 3 informes de investigación referentes a los cambios que el concepto de discapacidad intelectual ha sufrido a lo largo del tiempo. Para la gestión y clasificación de las referencias bibliográficas se ha utilizado el programa Mendeley.

\section{Resultados}

El enfoque psicoterapéutico en población con discapacidad intelectual es controvertido y la evidencia sobre las terapias psicológicas es limitada (Bhaumik et al., 2011). Aun así, el aumento de las demandas de proporcionar intervenciones psicológicas a este colectivo (Vereenooghe y Langdon, 2013) pone de relevancia la importancia de un tratamiento multimodal y no únicamente basado en la farmacología. Dosen (2007) también apoya un tratamiento integrativo (psicoterapia y farmacología) para la intervención en este colectivo, aunque prevalece la monoterapia, es decir, se pone énfasis en una única intervención. La razón que propone este autor sobre la utilización de un único tratamiento, es que en dicho colectivo predomina una visión reduccionista. Benson (2004) propone otros factores por los que el acceso de este colectivo a los servicios sanitarios es menor. Uno de ellos hace referencia a la visión de los profesionales de que los tratamientos psicológicos no son eficaces debido a los déficits cognitivos y, el otro, a las limitaciones verbales de estos individuos. Aun así, destacar la importancia de la combinación de diferentes intervenciones debido a los beneficios tanto para el cliente como para su entorno. Bhaumik et al. (2011) y Dosen (2007) también apoyan la combinación de varias intervenciones psicológicas dentro del plan de tratamiento, así como la creación de un plan individualizado para aumentar la eficacia de la intervención (Drury y Alim, 2014; Wilson, 2001). A continuación se describen qué terapias psicológicas se muestran como más eficaces en discapacidad intelectual y trastorno límite de la personalidad.

Destacar que la literatura existente sobre las intervenciones psicológicas en TLP y discapacidad intelectual es limitada y está basada en estudios de caso. Este hecho no significa que no existan casos pero no se suelen presentar con gran frecuencia en la práctica clínica. Aun así, este colectivo no está exento de presentar un TLP (Pridding y Procter, 2008; Wink et al., 2010). A pesar de estas limitaciones, se ha sugerido la terapia dialéctica conductual como la intervención que ha mostrado resultados positivos para reducir la sintomatología del trastorno límite de la personalidad en individuos con discapacidad intelectual (Crossland et al., 2017). Otro tipo de terapias estudiadas, como la psicoanalítica, ha demostrado en una muestra de 25 participantes, todos hombres con discapacidad intelectual (sin especificar la gravedad) y problemas com- 
portamentales, que existe una mejoría en la sintomatología conductual y que esta se mantiene a lo largo de 6 meses (Brown et al., 2011). Respecto a la terapia cognitivo conductual, muy utilizada ante varias condiciones incluidos los trastornos de personalidad (sin especificar el tipo), se ha demostrado potencialmente útil en mejorar la sintomatología en general (Brown et al., 2011). La terapia cognitiva no es muy apropiada para individuos con discapacidad intelectual, las dificultades verbales, las limitaciones de vocabulario, los problemas respecto al pensamiento abstracto y de memoria, dificultan la aplicación de la terapia (Brown et al., 2011).

Como se ha citado anteriormente, hasta el momento la terapia que ha aportado resultados favorables es la dialéctica conductual o la aplicación de algunas estrategias de dicha terapia para abordar la sintomatología que provoca malestar o riesgo para el individuo. Es por ello, que nos centraremos en explicar en qué consiste, la exposición de diversos casos encontrados en la literatura sobre su eficacia, las ventajas cuando se aplica al colectivo de discapacidad intelectual y qué dificultades se plantean.

La terapia dialéctica conductual se basa en un programa de tratamiento cognitivo-conductual muy sistematizado. Se trata de un modelo integrador y se compone de tres principios generales: la ciencia comportamental, la filosofía dialéctica y la práctica zen (mindfulness). Las funciones principales del programa son: potenciar capacidades del paciente, incrementar la motivación, potenciar la generalización, estructurar el ambiente y potenciar capacidades del terapeuta. $Y$ todo ello se lleva a cabo a través de sesiones individuales, que permiten disminuir/eliminar conductas suicidas o parasuicidas, disminuir/eliminar conductas que interfieran en la terapia, disminuir/eliminar conductas que interfieran con la calidad de vivir, adquisición de habilidades conductuales, aumento del respeto de uno mismo, y reducción de efectos de trastorno de estrés post traumático (traumas sexuales, físicos, emocionales); las sesiones grupales en formato psicoeducativo donde se trabajan las habilidades de toma de conciencia, regulación emocional, tolerancia al malestar y habilidades sociales; las consultas telefónicas de 24 horas con el terapeuta cuando aparecen situaciones de crisis y los encuentros de consulta entre terapeutas para aumentar la cooperación y contención entre sus miembros (Roscoe et al., 2015). Tras enmarcar en qué consiste la terapia, a continuación se exponen algunos estudios y sus resultados tras aplicar la terapia dialéctica conductual a individuos con discapacidad intelectual y TLP.

Wilson (2001) explica un modelo de cuatro fases para la gestión de las crisis que ha demostrado ser una herramienta extremadamente eficaz para el entrenamiento en el uso de enfoques terapéuticos para individuos con discapacidad intelectual y TLP. Se centra en las fluctuaciones comportamentales y las fases se dividen en cuatro estadios: el primero se denomina función óptima y su objetivo es que el comportamiento se mantenga en esta fase y las intervenciones utilizadas son enseñar y reforzar la conducta adecuada, mantener una estructura y enseñar y practicar habilidades de afrontamiento, calma y distracción; en el segundo se trata de detectar los antecedentes o factores precipitantes de un comportamiento inestable y la finalidad es volver a la primera fase con las habilidades citadas anteriormente y el mantenimiento de la estructura; el tercero es el estadio de crisis y el propósito es mantener una seguridad física y pasar a la cuarta fase, las dos intervenciones utilizadas son mantener seguro al paciente y observar señales de resolución de la crisis; finalmente en la resolución lo que se persigue es volver gradualmente a la primera fase a través de restaurar la estructura, validar sentimientos y la utilización de habilidades de afrontamiento, calma y distracción. El modelo también cuenta con una fase de entrenamiento para los profesionales que aplican el tratamiento: se les informa sobre la etiología y las características clínicas del TLP, las manifestaciones clínicas y etiológicas 
de los individuos a los que va dirigido el programa, la presentación del modelo de cuatro fases, que exista un compromiso por parte de los profesionales para garantizar la consecución de los objetivos y suministrar apoyo y revisión del progreso hacia los objetivos del tratamiento. Este mismo autor aplica su modelo a una mujer de 48 años con discapacidad intelectual de grado moderado que cumple criterios para TLP y con depresión mayor. Tras la aplicación del modelo, la paciente adquirió las habilidades adecuadas para regular su comportamiento y emociones, mejorando su calidad de vida y relaciones interpersonales e incrementado sus actividades vocacionales y lucrativas. Los seguimientos post intervención fueron al mes, a los dos, a los seis y a los diez meses y los episodios de autolesión, chillar o llorar disminuyeron y se mantuvieron. Pridding y Procter (2008) describen el mismo caso que Wilson (2001) y sugieren la importancia de realizar ciertas adaptaciones a las necesidades de los individuos al aplicar la terapia, para mejorar la comprensión y obtener resultados positivos. Benson (2004) expone el caso de una mujer joven (sin especificar la edad) con trastorno límite de la personalidad y discapacidad intelectual (sin especificar la gravedad) que recibía tratamiento psiquiátrico, psicológico e intervención conductual (sin especificar las estrategias empleadas). La integración de los diferentes servicios y profesionales, permitió disminuir las autolesiones y otro tipo de problemas conductuales. La no coordinación de los servicios se reflejó en un aumento del número de hospitalizaciones. Wink et al. (2010) explica tres casos de individuos con discapacidad intelectual leve y con TLP comórbido. El primero de ellos, se trata de un hombre de 25 años que tras el diagnostico de trastorno límite de la personalidad y con conductas verbales y físicas agresivas, arrebatos de ira y dosis elevadas de diferentes tipos de fármacos, se iniciaron diferentes intervenciones psicológicas. La primera fue la estructuración del ambiente terapéutico con profesionales formados en el ámbito. Los resultados a corto plazo permitieron la disminución de las reacciones emocionales como la autolesión e intento de suicidio, así como reacciones menos agresivas en el ambiente familiar. Posteriormente se introdujo un sistema de recompensas por las veces que no presentaba un comportamiento agresivo (destrucción de la propiedad, agresión física hacia los profesionales o llamar al servicio de emergencias) y la disminución de las hospitalizaciones. El resultado de las intervenciones fue el acceso a actividades agradables para el individuo y la mejora de las relaciones interpersonales tanto con amigos como con sus familiares. Para el mantenimiento de las actividades se estableció un sistema de fichas adaptado que permitió disminuir la frustración y ansiedad. Respecto al tratamiento farmacológico se redujo las dosis de ciertos fármacos sin presentar ningún empeoramiento de los síntomas. El segundo caso, se trata de un hombre de 37 años y previo al diagnóstico de TLP había recibido terapia conductual y tratamiento farmacológico sin éxito. Tras el diagnostico, se decidió cambiar el planteamiento de intervención para reducir las escasas habilidades de afrontamiento, las autoagresiones y su estado de ánimo bajo. La intervención fue de corte conductual para mejorar la baja autoestima; la estrategia fue aumentar las interacciones interpersonales en los momentos detectados como alto riesgo en sus comportamientos. Se llevaron a cabo conversaciones con los profesionales durante estas situaciones de alto riesgo, además de hablar sobre los progresos en su tratamiento y los aspectos positivos de su vida. Esta intervención permitió aumentar los sentimientos de cercanía y confianza con otros individuos. Otro tipo de estrategia utilizada fue el aprendizaje de técnicas de relajación para los síntomas de frustración y ansiedad. Todo lo citado, permitió mejorar el autocontrol, la sensación de poder dominar su angustia interior y la disminución de las autoagresiones. Respecto al tratamiento farmacológico se disminuyó las dosis de ciertos fármacos. El último individuo, una mujer de 21 años y con historia de abuso de drogas y maltrato por parte de su padre, había recibido terapia psicológica y tratamiento farmacológico para la depresión que presentaba sin éxito. Posteriormente, se le diagnosticó trastorno límite de la personalidad por la conducta autolesiva, agresiva, la impulsividad, los celos en su relación sentimental y la irritabilidad que presentaba. Las intervenciones psicológicas se realizaron dos días por semana y se centraron en aprender 
habilidades para disminuir los prejuicios y las reacciones a los estresores del ambiente. También se trabajó para hacerle ver que los profesionales estaban para ayudarla y no para castigarla o controlarla. A pesar de la ayuda recibida, la paciente continuaba presentando alguna dificultad conductual debido a un ambiente familiar inestable. Crossland et al. (2017) tomó una muestra de cuatro participantes en su investigación, tres mujeres y un hombre, con edades comprendidas entre los 24 y 48 años, con discapacidad intelectual leve y dificultades interpersonales y emocionales. Solo dos de los cuatro individuos cumplían criterios para un trastorno límite de la personalidad. Las intervenciones tuvieron una duración de 18 semanas, basadas en el entrenamiento de habilidades de la terapia dialéctica conductual. Los módulos a trabajar fueron los siguientes: mindfulness, tolerancia al malestar, regulación de emociones y habilidades interpersonales. Las mejoras se observaron en las relaciones interpersonales y aumento del bienestar para tres de los cuatro participantes a largo plazo (cuatro meses de seguimiento). Roscoe et al. (2015) realizó un estudio cualitativo con diez mujeres con discapacidad intelectual leve y un TLP comórbido, valorando sus puntos de vista, experiencias y comprensión sobre la aplicación de la terapia dialéctica conductual. Los resultados establecen que, en cuanto a la comprensión de la terapia, todas las participantes mostraron cierta dificultad en la comprensión de algunos conceptos, pero en general les fue beneficiosa para mejorar la sintomatología (habilidades de comunicación, interpersonales, de negociación y autocontrol). Otro factor que influyó en la terapia fue el número de pacientes en las sesiones de grupo; para algunas participantes fue positivo el hecho de compartir sus experiencias pero para otras fue una experiencia menos positiva; por último, destacar la importancia de la relación terapéutica y la motivación. Una de las estrategias en las que se observó más dificultad fue en la utilización de mindfulness; a pesar de ello, las participantes pudieron involucrarse y usarlas. Este hecho pone de relieve la importancia de la enseñanza de habilidades a través de métodos experienciales en lugar de medios teóricos o conceptuales, que pueden ser abstractos y difíciles para las personas con discapacidad intelectual.

Para finalizar, es conveniente citar las ventajas que supone aplicar la terapia dialéctica conductual en este colectivo. Se trata de un enfoque adecuado para potenciar las habilidades prácticas, los aspectos psicológicos y educativos, se basa en un modelo que utiliza un lenguaje positivo, no peyorativo y sin culpar al paciente y potencia el desarrollo del individuo basándose en valores como la asertividad, independencia, empoderamiento y autodefensa. A pesar de las ventajas, también se observan ciertos retos a mejorar. Teniendo en cuenta que algunas de las estrategias son de tipo cognitivo, hay que tener en cuenta aquellos individuos con habilidades de lectura y memoria pobres o ausentes. Algunas de las soluciones que se proponen se basan en la repetición de los conceptos, explicación breve y lenguaje comprensible, alentar a mantener respuestas sencillas (tipo bien o mal) y utilización de material visual. Otro desafío que se plantea, es que algunos individuos no estén preparados para participar en las sesiones grupales pudiendo desestructurar al resto de miembros; se plantea que la realización de los encuentros grupales se realice cuando sean necesarios para la persona y no por regla. Un tercer reto es que muchas de las habilidades trabajadas en las sesiones individuales o grupales se han de extrapolar a las vidas de los pacientes y es cuando pueden aparecer las dificultades. Como solución se recomienda que la generalización ha de planearse previamente, teniendo en cuenta cómo se llevara a cabo y detectar posibles obstáculos (Lew et al., 2006; Crossland et al., 2017; McNair et al., 2017). 


\begin{tabular}{|c|c|c|c|c|c|c|}
\hline Autor & Año & $\begin{array}{l}\text { Lugar de } \\
\text { realización }\end{array}$ & Muestra & Método & Tipo de terapia & Conclusiones \\
\hline Wilson & 2001 & $\begin{array}{l}\text { West Bay } \\
\text { Residential } \\
\text { Services, Warwick, } \\
\text { United Kingdom. }\end{array}$ & $\begin{array}{l}\text { Mujer de } 48 \\
\text { años con } \\
\text { discapacidad } \\
\text { intelectual } \\
\text { de grado } \\
\text { moderado } \\
\text { y trastorno } \\
\text { límite de la } \\
\text { personalidad. }\end{array}$ & $\begin{array}{l}\text { Descriptivo de un } \\
\text { caso. }\end{array}$ & \begin{tabular}{|l|} 
Modelo de cuatro \\
fases para el \\
tratamientos del \\
trastorno límite de \\
la personalidad \\
en individuos con \\
discapacidad \\
intelectual.
\end{tabular} & $\begin{array}{l}\text { El estudio sugiere que el } \\
\text { modelo ofrece una vía que } \\
\text { permite identificar los objetivos, } \\
\text { entrenar a los profesionales } \\
\text { y establecer intervenciones } \\
\text { basadas en la acción para } \\
\text { promover la autorregulación y } \\
\text { mejorar el comportamiento de } \\
\text { los individuos con trastorno } \\
\text { límite de la personalidad y } \\
\text { discapacidad intelectual. }\end{array}$ \\
\hline Benson & 2004 & $\begin{array}{l}\text { Nisonger Center, } \\
\text { Ohio State } \\
\text { University, Ohio, } \\
\text { United States. }\end{array}$ & $\begin{array}{l}\text { Estudios } \\
\text { realizados entre } \\
2003 \text { y } 2004 .\end{array}$ & $\begin{array}{l}\text { Revisión } \\
\text { sistemática. }\end{array}$ & $\begin{array}{l}\text { Analiza las } \\
\text { intervenciones } \\
\text { psicológicas en } \\
\text { individuos con } \\
\text { discapacidad } \\
\text { intelectual. }\end{array}$ & $\begin{array}{l}\text { Se observa que los estudios } \\
\text { predominantes en este } \\
\text { colectivo para valorar el tipo de } \\
\text { intervenciones psicológicas son } \\
\text { los estudios de caso. Este tipo } \\
\text { de investigaciones permite el } \\
\text { estudio de qué terapias son las } \\
\text { más utilizadas en gran variedad } \\
\text { de problemas. }\end{array}$ \\
\hline Lew et al. & 2006 & $\begin{array}{l}\text { The Bridge } \\
\text { of Central } \\
\text { Massachusetts, } \\
\text { Massachusetts, } \\
\text { United States. }\end{array}$ & $\begin{array}{l}8 \text { mujeres } \\
\text { entre } 25 \text { y } \\
61 \text { años con } \\
\text { discapacidad } \\
\text { intelectual } \\
\text { entre leve y } \\
\text { moderada y } \\
\text { diagnóstico } \\
\text { de trastorno } \\
\text { mental. } \\
\end{array}$ & Descriptivo. & $\begin{array}{l}\text { Analiza la } \\
\text { aplicación de la } \\
\text { terapia dialéctica } \\
\text { conductual en } \\
\text { individuos con } \\
\text { discapacidad } \\
\text { intelectual. }\end{array}$ & $\begin{array}{l}\text { Del estudio se concluye que los } \\
\text { comportamientos de riesgo de } \\
\text { las personas con discapacidad } \\
\text { intelectual, mejoran tras } \\
\text { la aplicación de la terapia } \\
\text { adaptada a sus necesidades. }\end{array}$ \\
\hline Dosen & 2007 & $\begin{array}{l}\text { Department } \\
\text { of Psychiatry, } \\
\text { Radboud } \\
\text { University, } \\
\text { Nijmegen, the } \\
\text { Netherlands. }\end{array}$ & $\begin{array}{l}\text { Hombre de } \\
21 \text { años con } \\
\text { discapacidad } \\
\text { intelectual de } \\
\text { grado leve con } \\
\text { episodios de } \\
\text { agresividad. } \\
\text { Mujer de } 21 \\
\text { años con } \\
\text { discapacidad } \\
\text { intelectual de } \\
\text { grado leve } \\
\text { acompañada } \\
\text { de problemas } \\
\text { de irritabilidad, } \\
\text { agresividad y } \\
\text { oposición. }\end{array}$ & $\begin{array}{l}\text { Descriptivo de un } \\
\text { caso. }\end{array}$ & $\begin{array}{l}\text { Tratamiento } \\
\text { multidimensional } \\
\text { aplicado a las } \\
4 \text { dimensiones } \\
\text { (biológico, } \\
\text { psicológico, } \\
\text { de desarrollo y } \\
\text { social). }\end{array}$ & $\begin{array}{l}\text { Los resultados apoyan a que la } \\
\text { intervención multidimensional, } \\
\text { teniendo en cuenta las } 4 \\
\text { dimensiones y utilizando } \\
\text { diferentes modelos terapéuticos } \\
\text { (no se especifican), tiene } \\
\text { efectos positivos para restaurar } \\
\text { el bienestar mental de los } \\
\text { individuos con discapacidad } \\
\text { intelectual. A todo ello, la } \\
\text { necesidad de instruir a los } \\
\text { profesionales sobre cómo } \\
\text { aplicar los tratamientos es } \\
\text { fundamental y de la adaptación } \\
\text { de los modelos terapéuticos } \\
\text { a las características de estos } \\
\text { individuos. }\end{array}$ \\
\hline
\end{tabular}




\begin{tabular}{|c|c|c|c|c|c|c|}
\hline Autor & Año & $\begin{array}{l}\text { Lugar de } \\
\text { realización }\end{array}$ & Muestra & Método & Tipo de terapia & Conclusiones \\
\hline $\begin{array}{l}\text { Pridding y } \\
\text { Procter }\end{array}$ & 2008 & $\begin{array}{l}\text { St Vincent's } \\
\text { Hospital, } \\
\text { Melbourne, } \\
\text { Australia. }\end{array}$ & & $\begin{array}{l}\text { Revisión } \\
\text { sistemática. }\end{array}$ & $\begin{array}{l}\text { Analiza las } \\
\text { implicaciones } \\
\text { mentales de las } \\
\text { personas con } \\
\text { discapacidad } \\
\text { intelectuales y un } \\
\text { trastorno de la } \\
\text { personalidad. }\end{array}$ & $\begin{array}{l}\text { Individuos con trastorno de la } \\
\text { personalidad y discapacidad } \\
\text { intelectual son más } \\
\text { susceptibles a recibir menos } \\
\text { atención sanitaria debido al } \\
\text { estigma social y las dificultades } \\
\text { en asesoramiento. A todo } \\
\text { ello se suma la escasez de } \\
\text { tratamientos adaptados a sus } \\
\text { necesidades. }\end{array}$ \\
\hline Wink et al. & 2010 & $\begin{array}{l}\text { Department } \\
\text { of Psychiatry, } \\
\text { Indiana School } \\
\text { of Medicine, } \\
\text { Indianapolis, } \\
\text { United States. }\end{array}$ & $\begin{array}{l}2 \text { hombres y } 1 \\
\text { mujer entre } 21 \\
\text { y } 37 \text { años con } \\
\text { discapacidad } \\
\text { intelectual leve } \\
\text { y trastorno } \\
\text { límite de la } \\
\text { personalidad. }\end{array}$ & $\begin{array}{l}\text { Descriptivo de un } \\
\text { caso. }\end{array}$ & $\begin{array}{l}\text { Analiza las } \\
\text { intervenciones } \\
\text { psicológicas en } \\
\text { tres casos de } \\
\text { individuos con } \\
\text { discapacidad } \\
\text { intelectual y } \\
\text { trastorno límite de } \\
\text { la personalidad. }\end{array}$ & $\begin{array}{l}\text { Se concluye que un buen } \\
\text { diagnóstico del trastorno límite } \\
\text { de personalidad permite guiar } \\
\text { las diferentes intervenciones } \\
\text { posteriores. Tras la aplicación } \\
\text { de la terapia psicológica y } \\
\text { farmacológica se observa } \\
\text { que hay una mejoría de la } \\
\text { sintomatología y del bienestar } \\
\text { de los pacientes. }\end{array}$ \\
\hline $\begin{array}{l}\text { Bhaumik } \\
\text { et al. }\end{array}$ & 2011 & $\begin{array}{l}\text { Faculty of Learning } \\
\text { Disability of the } \\
\text { Royal College } \\
\text { of Psychiatrists } \\
\text { and Honorary } \\
\text { Senior Lecturer at } \\
\text { the University of } \\
\text { Leicester, United } \\
\text { Kingdom. } \\
\text { Leicestershire } \\
\text { Partnership NHS } \\
\text { Trust, United } \\
\text { Kingdom. } \\
\text { Department of } \\
\text { Child Psychiatry } \\
\text { at the Christian } \\
\text { Medical College, } \\
\text { Vellore, India. }\end{array}$ & & Descriptivo. & $\begin{array}{l}\text { Terapia } \\
\text { conductual, } \\
\text { cognitivo- } \\
\text { conductual y } \\
\text { psicoanalítica. }\end{array}$ & $\begin{array}{l}\text { Teniendo en cuenta que } \\
\text { la investigación sobre los } \\
\text { tratamientos psicológicos en } \\
\text { individuos con discapacidad } \\
\text { intelectual es limitada, se } \\
\text { observa que el análisis } \\
\text { funcional de la conducta se } \\
\text { ha visto que es eficaz dentro } \\
\text { del modelo conductual, } \\
\text { respecto a la terapia cognitivo- } \\
\text { conductual tiene evidencia } \\
\text { tanto a corto como a largo } \\
\text { plazo en sintomatología } \\
\text { ansiosa, depresiva o control } \\
\text { de la ira y la psicoanalítica } \\
\text { se ha visto adecuada para } \\
\text { mejorar la autoestima, pero } \\
\text { con un inconveniente respecto } \\
\text { a los déficits en habilidades } \\
\text { comunicativas de estos } \\
\text { individuos. }\end{array}$ \\
\hline Brown et al. & 2011 & $\begin{array}{l}\text { NHS Lothian, } \\
\text { United Kingdom. } \\
\text { Edinburgh Napier } \\
\text { University, United } \\
\text { Kingdom. }\end{array}$ & & $\begin{array}{l}\text { Revisión } \\
\text { sistemática. }\end{array}$ & $\begin{array}{l}\text { Terapia } \\
\text { psicodinámica } \\
\text { individual y } \\
\text { grupal, cognitivo- } \\
\text { conductual, } \\
\text { counselling y } \\
\text { terapia sistémica. }\end{array}$ & $\begin{array}{l}\text { La revisión sugiere que } \\
\text { la investigación sobre las } \\
\text { intervenciones psicológicas en } \\
\text { individuos con funcionamiento } \\
\text { intelectual límite está } \\
\text { aumentando, pero aún se } \\
\text { requiere más estudios. La } \\
\text { adaptación de las terapias en } \\
\text { función de las necesidades } \\
\text { de los individuos debe ser } \\
\text { necesaria para obtener unos } \\
\text { resultados favorables. }\end{array}$ \\
\hline
\end{tabular}




\begin{tabular}{|c|c|c|c|c|c|c|}
\hline Autor & Año & $\begin{array}{l}\text { Lugar de } \\
\text { realización }\end{array}$ & Muestra & Método & Tipo de terapia & Conclusiones \\
\hline $\begin{array}{l}\text { Vereenooghe } \\
\text { y Langdon }\end{array}$ & 2013 & $\begin{array}{l}\text { Department of } \\
\text { Psychological } \\
\text { Sciences, Norwich } \\
\text { Medical School, } \\
\text { University of East } \\
\text { Anglia, Norwich, } \\
\text { United Kingdom. } \\
\text { Broadland Clinic, } \\
\text { Hertfordshire } \\
\text { Partnership } \\
\text { University NHS } \\
\text { Foundation Trust } \\
\text { Norfolk, United } \\
\text { Kingdom. }\end{array}$ & & $\begin{array}{l}\text { Revisión } \\
\text { sistemática y meta } \\
\text { análisis (solo se } \\
\text { ha centrado la } \\
\text { información en la } \\
\text { parte cualitativa). }\end{array}$ & $\begin{array}{l}\text { Analiza la } \\
\text { eficacia de los } \\
\text { tratamientos } \\
\text { psicológicos en } \\
\text { individuos con } \\
\text { discapacidad } \\
\text { intelectual y } \\
\text { trastorno mental } \\
\text { comórbido a nivel } \\
\text { general. }\end{array}$ & $\begin{array}{l}\text { El estudio establece que la } \\
\text { terapia cognitivo-conductual } \\
\text { es adecuada para cierta } \\
\text { sintomatología como la ira } \\
\text { o depresión en individuos } \\
\text { con discapacidad intelectual. } \\
\text { Aun así, se requiere de más } \\
\text { investigación sobre la eficacia } \\
\text { de otras intervenciones } \\
\text { psicológicas. }\end{array}$ \\
\hline Drury y Alim & 2014 & $\begin{array}{l}\text { Southwark } \\
\text { Psychological } \\
\text { Therapies Service, } \\
\text { South London and } \\
\text { Maudsley NHS } \\
\text { Foundation Trust, } \\
\text { London, United } \\
\text { Kingdom. } \\
\text { Mental Health } \\
\text { in Learning } \\
\text { Disabilities } \\
\text { Lewisham } \\
\text { Psychology, } \\
\text { South London and } \\
\text { Maudsley NHS } \\
\text { Foundation Trust, } \\
\text { London, United } \\
\text { Kingdom. }\end{array}$ & $\begin{array}{l}\text { Hombre de } \\
50 \text { años con } \\
\text { discapacidad } \\
\text { intelectual de } \\
\text { grado leve, con } \\
\text { rasgos autistas } \\
\text { y estado de } \\
\text { ánimo bajo. }\end{array}$ & $\begin{array}{l}\text { Descriptivo de un } \\
\text { caso. }\end{array}$ & $\begin{array}{l}\text { Analiza la } \\
\text { importancia de } \\
\text { la combinación } \\
\text { de diferentes } \\
\text { modelos } \\
\text { psicológicos en } \\
\text { tratamiento de } \\
\text { individuos con } \\
\text { discapacidad } \\
\text { intelectual y } \\
\text { trastorno mental. }\end{array}$ & $\begin{array}{l}\text { Se ha observado que el } \\
\text { acercamiento integrado no } \\
\text { solo ha permitido al cliente } \\
\text { desarrollar sus habilidades } \\
\text { en la solución de problemas } \\
\text { y la toma de perspectivas } \\
\text { diferentes, sino también ha } \\
\text { generado que el individuo } \\
\text { tenga un papel más activo } \\
\text { en todas aquellas decisiones } \\
\text { relacionadas con su vida. }\end{array}$ \\
\hline Roscoe et al. & 2015 & $\begin{array}{l}\text { Bangor University, } \\
\text { United Kingdom. } \\
\text { Mersey Care NHS } \\
\text { Trust, United } \\
\text { Kingdom. } \\
\text { Warwick University, } \\
\text { United Kingdom. }\end{array}$ & $\begin{array}{l}10 \text { mujeres } \\
\text { entre } 19 \text { y } \\
57 \text { años con } \\
\text { discapacidad } \\
\text { intelectual leve } \\
\text { y un trastorno } \\
\text { límite de la } \\
\text { personalidad. }\end{array}$ & $\begin{array}{l}\text { Análisis } \\
\text { fenomenológico } \\
\text { interpretativo (IPA). }\end{array}$ & $\begin{array}{l}\text { Analiza las } \\
\text { opiniones y } \\
\text { puntos de vista } \\
\text { a través de } \\
\text { entrevistas sobre } \\
\text { su experiencia } \\
\text { con la terapia } \\
\text { dialéctica } \\
\text { conductual. }\end{array}$ & $\begin{array}{l}\text { Los resultados destacan la } \\
\text { importancia de una adaptación } \\
\text { de la estructura de la terapia } \\
\text { a las necesidades de los } \\
\text { participantes. Uno de los } \\
\text { factores que jugó un rol } \\
\text { fundamental fue la relación } \\
\text { terapéutica, influyendo en la } \\
\text { motivación, compromiso y la } \\
\text { participación. }\end{array}$ \\
\hline $\begin{array}{l}\text { Crossland } \\
\text { et al. }\end{array}$ & 2017 & $\begin{array}{l}\text { Learning Disability } \\
\text { Services, Berkshire } \\
\text { Healthcare NHS } \\
\text { Foundation Trust, } \\
\text { Reading, United } \\
\text { Kingdom. }\end{array}$ & $\begin{array}{l}4 \text { participantes } \\
\text { (3 mujeres y } 1 \\
\text { hombre) con } \\
\text { discapacidad } \\
\text { intelectual } \\
\text { leve y solo } \\
\text { dos de ellos } \\
\text { con trastorno } \\
\text { límite de la } \\
\text { personalidad. }\end{array}$ & $\begin{array}{l}\text { Entrevistas } \\
\text { cualitativas y } \\
\text { análisis temático } \\
\text { de las entrevistas. }\end{array}$ & $\begin{array}{l}\text { Analiza la } \\
\text { eficacia de la } \\
\text { aplicación de la } \\
\text { terapia dialéctica } \\
\text { conductual a } \\
\text { individuos con } \\
\text { discapacidad y } \\
\text { trastorno límite de } \\
\text { la personalidad. }\end{array}$ & $\begin{array}{l}\text { Se observó la mejora en } \\
\text { diferentes áreas para } 3 \text { de los } 4 \\
\text { participantes y estas mejorías } \\
\text { se mantuvieron a largo plazo (4 } \\
\text { meses posteriores). }\end{array}$ \\
\hline
\end{tabular}




\begin{tabular}{|c|c|c|c|c|c|c|}
\hline Autor & Año & $\begin{array}{l}\text { Lugar de } \\
\text { realización }\end{array}$ & Muestra & Método & Tipo de terapia & Conclusiones \\
\hline Mcnair et al. & 2017 & \begin{tabular}{|l|} 
Cheshire and Wirral \\
Partnership NHS \\
Foundation Trust, \\
Cheshire, United \\
Kingdom. \\
School of \\
Psychology, Cardiff \\
University, Cardiff, \\
United Kingdom.
\end{tabular} & $\begin{array}{l}7 \text { estudios entre } \\
1980 \text { y } 2013 .\end{array}$ & $\begin{array}{l}\text { Revisión } \\
\text { sistemática. }\end{array}$ & \begin{tabular}{|l|} 
Analiza la \\
literatura \\
existente sobre \\
la utilización \\
de las terapias \\
psicológicas en \\
individuos con \\
discapacidad \\
intelectual; y \\
específicamente \\
la dialéctica \\
conductual en \\
personas con \\
trastorno límite de \\
la personalidad.
\end{tabular} & $\begin{array}{l}\text { Los resultados apuntan que } \\
\text { la utilización de la terapia } \\
\text { dialéctico conductual como un } \\
\text { todo y las sesiones de grupo } \\
\text { de habilidades específicamente } \\
\text { pueden ser adaptadas al } \\
\text { colectivo. Aun así, es necesaria } \\
\text { más investigación en el ámbito. }\end{array}$ \\
\hline
\end{tabular}

Fuente: elaboración propia.

\section{Conclusiones}

El objetivo de la presente revisión bibliográfica es examinar la eficacia y qué tipos de intervenciones se llevan a cabo en individuos con discapacidad intelectual y trastorno límite de la personalidad. Tras la revisión de la literatura, la aplicación de las estrategias que componen la terapia dialéctica conductual en este colectivo ha demostrado una reducción en la frecuencia y severidad en el comportamiento parasuicida, las hospitalizaciones, las interferencias en terapia y la mejora en el ajuste emocional. Las intervenciones basadas en la orientación psicodinámica sugieren una mejoría en problemas conductuales en individuos con discapacidad intelectual, pero no existe evidencia en el abordaje del trastorno límite de personalidad en dicho colectivo. Otra terapia que permite mejorar la sintomatología ante varias condiciones, incluidos los trastornos de personalidad (sin especificar cuál) es la cognitivo conductual, pero al igual que la psicodinámica no existen estudios aplicados en TLP y discapacidad intelectual. Finalmente, como tratamiento estudiado en población general, pero no recomendado para población con discapacidad intelectual, se encuentra la terapia cognitiva, ya que presentan dificultades en la utilización del lenguaje y pensamiento abstracto, entre otras.

Tal y como podemos observar, y según los estudios encontrados en la literatura, la terapia que mejores resultados ha demostrado para disminuir la sintomatología del trastorno límite de la personalidad en individuos con discapacidad intelectual es la dialéctica conductual con adaptaciones. Aun así, los resultados encontrados se han de tomar con precaución, ya que se requiere de más evidencia con metodología más rigurosa para afirmar que la terapia dialéctica conductual se trata de una intervención bien establecida. Destacar la importancia de tener en cuenta la gravedad de la discapacidad intelectual, a la hora de aplicar los tratamientos. Por último, señalar que el acceso de esta población a los recursos de salud mental para beneficiarse de los tratamientos disponibles, permitiría mejorar el funcionamiento del día a día y aliviar la sintomatología. 
Finalmente, se exponen una serie de limitaciones en este ámbito para así mejorar las futuras investigaciones sobre la efectividad de las terapias psicológicas en individuos con discapacidad intelectual y TLP:

- La escasez de literatura en este ámbito. De forma general el colectivo de individuos con discapacidad intelectual con un trastorno límite de la personalidad, es poco frecuente en la práctica clínica. Es por ello que las investigaciones son muy limitadas.

- Gran parte de las investigaciones se basan en estudios de caso. Consecuentemente hay una falta de control en la influencia de las variables extrañas que puede estar afectando a los resultados. La utilización de una metodología más rigurosa para el control de estas variables (aleatorización, grupo control, doble ciego) permitiría obtener mejores resultados.

- La falta de investigación respecto a las intervenciones en función del nivel de discapacidad intelectual. Determinar el nivel de afectación de la discapacidad intelectual es importante, ya que individuos con un nivel leve presentan unos déficits y necesidades diferentes a los de un nivel más severo. Y, por lo tanto, las intervenciones deberán ajustarse a su nivel de afectación. 
Referencias bibliográficas

AAIDD (2011): Discapacidad intelectual. Definición, clasificación y sistemas de apoyo social. Madrid: Alianza Editorial.

Benson, B. (2004): "Psychological interventions for people with intellectual disability and mental health problems". Current Opinion in Psychiatry, 17 (5): 353-357.

Bhaumik, S. et al. (2011): "Psychological treatments in intellectual disability: the challenges of building a good evidence base". The British Journal of Psychiatry, 198: 428-430.

Brown, M. et al. (2011): "A review of the literature relating to psychological interventions and people with intellectual disabilities: issues for research, policy, education and clinical practice". Journal of Intellectual Disabilities, 15 (1): 31-45.

Costello, H. y Bouras, N. (2006): "Assessment of mental health problems in people with intellectual disabilities". The Israel Journal of Psychiatry and Related Sciences, 43 (4): 241-51.

Crossland, T. et al. (2017): "Outcomes and experiences of an adapted dialectic behavior therapy skills training group for people with intellectual disabilities”. British Journal of Learning Disabilities, 45: 208-2016.

Di Nuovo, S. F. y Buono, S. (2007): "Psychiatric syndromes comorbid with mental retardation: differences in cognitive and adaptive skills". Journal of Psychiatric Research, 41 (9): 795-800.

Dosen, A. (2007): "integrative treatment in persons with intelectual disability and mental health problems". Journal of Intellectual Disability Research, 15 (1): 66-74.

Drury, H. y Alim, N. (2014): "Integrative psychotherapy in intelectual disabilities: using cognitive-behavioural and psychodynamic formulations". Advances in mental health and intellectual disabilities, 8 (3): 197-209.

Kerker, B. et al. (2004): "Mental health disorders among individuals with mental retardation: challenges to accurate prevalence estimates". Public Health Reports, 119 (4): 409-17.

Lew, M. et al. (2006): "Dialectical Behavior Therapy (DBT) for individuals with intellectual disabilities: a program description". Mental Health Aspects of Developmental Disabilities, 9 (1): 1-13.

Martorell A. et al. (2009): "Mental health in adults with mild and moderate intellectual disabilities: the role of recent life events and traumatic experiences across the life span". The Journal of Nervous and Mental Disease, 197 (3): $182-186$.

McNair, L. et al. (2017): "Dialectical Behaviour Therapy [DBT] with people with intellectual disabilities: a systematic review and narrative analysis". Journal of Applied Research in Intellectual Disabilities, 30 (5): 787-804.

Pridding, A., y Procter, N. (2008): "A systematic review of personality disorder amongst people with intellectual disability with implications for the mental health nurse practitioner". Journal of Clinical Nursing, 17 (21): 2811-9.

Roscoe, P. et al. (2015): "Dialectical Behaviour Therapy in an inpatient unit for women with a learning disability: service user's perspectives". Journal of Intellectual Disabilities, 1-18.

Rush, K. et al. (2004): "Assessing psychopathology in individuals with developmental disabilities”. Behavior Modification, 28 (5): 621-637. 
Schalock, R. et al. (2007): "El nuevo concepto de retraso mental: comprendiendo el cambio al término de discapacidad intelectual”. Revista Española de Discapacidad Intelectual, 38 (4): 5-20.

Turygin, N. et al. (2014): "Prevalence of co-occurring disorders in a sample of adults with mild and moderate intellectual disabilities who reside in a residential treatment setting". Research in Developmental Disabilities, 35 (7): $1802-1808$.

Vereenooghe, L. y Langdon, P. (2013): "psychological therapies for people with intellectual disabilities: a systematic review and meta-analysis". Research in Developmental Disabilities, 34: 4085-4102.

Wieland, J. et al. (2015): "The prevalence of personality disorders in psychiatric outpatients with borderline intellectual functioning: comparison with outpatients from regular mental health care and outpatients with mild intellectual disabilities". Nordic Journal of Psychiatry, 69 (8): 599-604.

Wieland, J. et al. (2014): "psychiatric disorders in outpatients with borderline intellectual functioning: comparison with both outpatients from regular mental health care and outpatients with mild intellectual disabilities". Canadian Journal of Psychiatry, 59 (4): 213-219.

Wilson, S. (2001): "A four-stage model for management of borderline personality disorder in people with mental retardation". Mental Health Aspects of Developmental Disabilities, 4 (2): 68-76.

Wink, L. et al. (2010): "Co-morbid intellectual disability and borderline personality disorder: a case series". Psychiatry Interpersonal and Biological Processes, 73 (3): 277-287.

Whitaker, S. y Read, S. (2006): "The prevalence of psychiatric disorders among people with intellectual disabilities: an analysis of the literature". Journal of Applied Research in Intellectual Disabilities, 19: 330-345. 